(per 10,000 births) in the neighboring country of Iran and 4.9 (per 10,000 births) in Hungary. These figures show that an "outbreak of NTDs" is seemingly being occurred in the Iraqi region of Al-Ramadi in the west of the country. The rate of NTDs in this region of Iraq is about 2.6, 3.4, 3.8, 4.7 and 6.7 times higher than that of reported from Cuba, Norway, China, Iran and Hungary, respectively. It is also 3.2 times higher than that of estimated/expected for the global population.

Comments The very high occurrence of NTDs in this Iraqi region indicates that there is an urgent need for an action by regional health authorities and international agencies to the control of this outbreak in the area.

\section{THE PATTERN OF NEURAL TUBE DEFECTS IN A HIGHLY ENDOGAMOUS SOCIETY: 25 YEAR INCIDENCE TRENDS}

doi:10.1136/archdischild-2012-302724.0620

'B Kurdi, ${ }^{2} \mathrm{~A}$ Bener. 'Department of Paediatrics, Hamad Medical Center; ${ }^{2}$ Public Health, Weill Cornell Medical College \& Hamad Medical Corporation, Doha, Oatar

Objective The aim of this study is to determine the incidence and trends of NTD over a period of 25 years in the State of Qatar.

Design This is a retrospective hospital based cohort study.

Setting The survey was carried out in the main territory hospital in the State of Qatar.

Subjects and methods The study was conducted from 1985 to 2009 with a total of 302,049 newborns at the Women's Hospital in Qatar screened for NTD. NTD were defined according to the International Classification of Diseases, Tenth Revision (ICD-10). Study parameters included age, gender, ethnicity, parental consanguinity, and residential area.

Results The combined prevalence of NTD (total myelomeningocele and anencephaly) during the 25 years period was 1.09 per 1000 births. The prevalence of anencephaly was 0.36 per 1000 births. There were 131 (42.1\%) males and 180 (57.9\%) female newborns with NTD, corresponding to incidence of 0.899 and 1.289 per 1000 in male and female newborns, respectively. This difference was significant $(p<0.001)$. Consanguinity was seen in $36.7 \%$ of the parents.

Conclusion The present study revealed that Qatar has a relatively low incidence of NTD which is comparable to neighboring countries in the Arabian Gulf region. High rates of consanguinity and lack of periconceptual folic acid intake among mothers appear to be the major factors contributing to NTD in Qatar.

\section{1 \\ POLYMORPHIC LENGTH OF FOXE1 ALANINE STRETCH IN ISOLATED CLEFT LIP AND/OR PALATE}

doi:10.1136/archdischild-2012-302724.0621

${ }^{1,2} \mathrm{HP}$ Hsiao, ${ }^{3} \mathrm{HH}$ Lin, ${ }^{4} \mathrm{CY}$ Chen, ${ }^{2} \mathrm{BH}$ Chen, ${ }^{2} \mathrm{MC}$ Chao. ${ }^{1}$ Department of Paediatrics, Kaohsiung Municipal Hsiao-Kang Hospital; '2Department of Paediatrics, Kaohsiung Medical University Hospital, Kaohsiung Medical University; ' ${ }^{3}$ Department of Laboratory Medicine, Kaohsiung Municipal Hsiao-Kang Hospital; ${ }^{4}$ Department of Dentistry, E-Da Hospital, I-Shou University, Kaohsiung, Taiwan R.O.C.

Background and Aim Isolated cleft lip with/or cleft palate (CL/P) is a common complex birth defect that varies in prevalence with Asian and Amerindian ancestry having the highest rates. While several genes had significant association to the CL/P, the FOXE1 gene is involved in embryonic formation and is expressed in the secondary palate epithelium in human fetus. Since the first description of FOXE1 mutation found in cases of cleft palate, thyroid agenesis and choanal atresia, Moreno had conducted a genome-wide scan for $\mathrm{CL} / \mathrm{P}$ patients and confirmed the highly linkage to the $9 \mathrm{q} 23-33$, which resided the potential FOXE1. Herein, we are trying to investigate the isolated CL/P in Taiwan to see whether the polymorphic length of FOXE1 play an important role in the palatogenesis.
Method Eighty patients with isolated CL/PCL/P and one hundred controls were recruited in the study. Genomic DNA was amplified by PCR the amplicons containing polyalanine tract (234 to $258 \mathrm{bp}$; 11-19 alanines) were purified, then directly sequenced.

Results and conclusion The 14/14 genotype in polymorphic alanine stretch was most frequent both in cases (98.7\%) and in controls $(98.0 \%)$, whereas the heterozygous $14 / 16$ accounted for one case and two controls. The 14 alanine strech accounts for the major allele frequency of the polymorphic length in FOXE1 which consists of the high frequency in previous report from Japan. Although CL/P patients was linked to FOXE1, the polymorphic FOXE1 alanine stretch has no association with isolated CL/P in Taiwan. It appears to reflect the heterogeneity in formation of $\mathrm{CL} / \mathrm{P}$, perhaps a population difference.

\section{CONGENITAL CYSTIC ADENOMATOID MALFORMATION: IS IT ALWAYS BAD NEWS?}

doi:10.1136/archdischild-2012-302724.0622

${ }^{1} \mathrm{~K}$ Yadav, 'D Vasudev, ${ }^{2} \mathrm{~J}$ Budd, 'A 0 Akuma. 'University Hospitals of Leicester; ${ }^{2}$ University of Leicester, Leicester, UK

Aim To evaluate whether conservative approach to management of asymptomatic neonates with antenatal diagnosis of congenital cystic adenomatoid malformation of the lungs (CCAM) is safe and appropriate.

Method We undertook a retrospective review of all cases with antenatal diagnosis of CCAM from 2004-2010 in a lead perinatal centre. Pertinent data was extracted and the outcome and management of all affected infants were reviewed up to 1year of age.

Results Twenty-six pregnancies were complicated with CCAM. Three pregnancies were excluded: two terminated and one fetus had bronchogenic cyst. Twenty-three singleton pregnancies were included in the study. There was a threefold increase in CCAM in the last two years of the study as compared to the first two years. CCAM lesions underwent complete resolution in four $(17 \%)$ fetuses, partial resolution in $12(52 \%)$, remained static in four $(17 \%)$ and increased in one (4\%) fetus. Eleven (48\%) fetuses did not have any associated complications. 15 (65\%) infants were asymptomatic at birth and eight (35\%) were symptomatic. In two symptomatic infants respiratory distress resolved spontaneously. Three symptomatic infants had surgery and one died. A further three symptomatic preterm infants died due to extreme prematurity. All the surviving, non-operated infants: 17 (74\%) were followed up and none required hospitalization or surgical interventions in infancy.

Conclusions Our study shows that conservative management of asymptomatic infants with CCAM; consisting of symptoms surveillance, radiological investigations and consideration for surgery if symptoms arise and persist is safe and may be more appropriate to elective surgery in infancy.

\section{GASTROSCHISIS TRANSFERS CONDUCTED BY THE WEST MIDLANDS NEONATAL TRANSFER SERVICE}

doi:10.1136/archdischild-2012-302724.0623

${ }^{1} \mathrm{~A}$ Shenvi, ${ }^{2} \mathrm{~J}$ Harrison, ${ }^{1,2} \mathrm{~A}$ Philpott, ${ }^{1,2} \mathrm{~A}$ Dhillon, ${ }^{1} \mathrm{SV}$ Rasiah. 'Department of Neonatal Medicine; ${ }^{2}$ West Midlands Neonatal Transfer Service, Birmingham Women's Hospital, Birmingham, UK

Background and Aims Gastroschisis has an incidence of approx 3 per $10,000^{1}$ with an increasing trend in the UK. ${ }^{2}$ Gastroschisis requires immediate postnatal surgical care. Within the West Midlands, newborns with gastroschisis are resuscitated and transferred by WMNTS to a surgical centre. Network guidelines suggest completion of transfers within 4 hours of birth.

Methods Retrospective review of gastroschisis transfers conducted by the WMNTS from Jan 2008 to Dec 2011. 